\title{
How big is an OMI pixel?
}

\author{
Martin de Graaf ${ }^{1,3}$, Holger Sihler ${ }^{2}$, Lieuwe G. Tilstra ${ }^{3}$, and Piet Stammes ${ }^{3}$ \\ ${ }^{1}$ Delft University of Technology, Delft, the Netherlands \\ ${ }^{2}$ Max-Planck-Institut für Chemie, Mainz, Germany \\ ${ }^{3}$ Royal Netherlands Meteorological Institute, De Bilt, the Netherlands
}

Correspondence to: Martin de Graaf (graafdem@knmi.nl)

Received: 29 February 2016 - Published in Atmos. Meas. Tech. Discuss.: 24 March 2016

Revised: 30 June 2016 - Accepted: 7 July 2016 - Published: 4 August 2016

\begin{abstract}
The Ozone Monitoring Instrument (OMI) is a push-broom imaging spectrometer, observing solar radiation backscattered by the Earth's atmosphere and surface. The incoming radiation is detected using a static imaging CCD (charge-coupled device) detector array with no moving parts, as opposed to most of the previous satellite spectrometers, which used a moving mirror to scan the Earth in the acrosstrack direction. The field of view (FoV) of detector pixels is the solid angle from which radiation is observed, averaged over the integration time of a measurement. The OMI FoV is not quadrangular, which is common for scanning instruments, but rather super-Gaussian shaped and overlapping with the FoV of neighbouring pixels. This has consequences for pixel-area-dependent applications, like cloud fraction products, and visualisation.

The shapes and sizes of OMI FoVs were determined preflight by theoretical and experimental tests but never verified after launch. In this paper the OMI FoV is characterised using collocated MODerate resolution Imaging Spectroradiometer (MODIS) reflectance measurements. MODIS measurements have a much higher spatial resolution than OMI measurements and spectrally overlap at $469 \mathrm{~nm}$. The OMI FoV was verified by finding the highest correlation between MODIS and OMI reflectances in cloud-free scenes, assuming a 2-D super-Gaussian function with varying size and shape to represent the OMI FoV. Our results show that the OMPIXCOR product $75 \mathrm{FoV}$ corner coordinates are accurate as the full width at half maximum (FWHM) of a super-Gaussian FoV model when this function is assumed. The softness of the function edges, modelled by the super-Gaussian exponents, is different in both directions and is view angle dependent.
\end{abstract}

The optimal overlap function between OMI and MODIS reflectances is scene dependent and highly dependent on time differences between overpasses, especially with clouds in the scene. For partially clouded scenes, the optimal overlap function was represented by super-Gaussian exponents around 1 or smaller, which indicates that this function is unsuitable to represent the overlap sensitivity function in these cases. This was especially true for scenes before 2008, when the time differences between Aqua and Aura overpasses was about $15 \mathrm{~min}$, instead of $8 \mathrm{~min}$ after 2008. During the time between overpasses, clouds change the scene reflectance, reducing the correlation and influencing the shape of the optimal overlap function.

\section{Introduction}

The Ozone Monitoring Instrument (OMI) (Levelt et al., 2006) was launched in 2004 on board the Aura satellite, in a polar, sun-synchronous orbit at approximately $705 \mathrm{~km}$ altitude, with a local Equator-crossing time of 13:45 (ascending node). Its main objective is to monitor trace gases in the Earth's atmosphere, especially ozone. It was built as the successor to the ESA instruments Global Ozone Monitoring Experiment (GOME) (Burrows et al., 1999) and Scanning Imaging Absorption Spectrometer for Atmospheric Chartography (SCIAMACHY) (Bovensmann et al., 1999), and NASA's Total Ozone Mapping Spectrometer (TOMS) instruments (e.g. Fleig et al., 1986; Bhartia et al., 2013). GOME and SCIAMACHY were the first space-borne hyperspectral instruments, measuring the complete spectrum from the ultraviolet (UV) to shortwave infrared (SWIR) wavelength range with a relatively high spectral resolution (typically $0.2-$ 
$1.5 \mathrm{~nm}$ ), from which multiple trace gases, clouds, and aerosol parameters can be retrieved simultaneously. TOMS instruments have been monitoring the ozone column at a relatively high spatial resolution $\left(50 \times 50 \mathrm{~km}^{2}\right)$ with daily global coverage since 1978. OMI was designed to combine those functions and measure the complete spectrum from the UV to the visible wavelength range (up to $500 \mathrm{~nm}$ ) with a high spatial resolution and daily global coverage. To this end, the imaging optics were completely redesigned.

Instead of a rotating mirror, in OMI a twodimensional CCD (charge-coupled device) detector array $(780 \times 576$ pixels $)$ is used to map the incoming radiation in the across-track and wavelength dimensions simultaneously. A swath of about $2600 \mathrm{~km}$ in the across-track direction is imaged along one dimension of the detector array. Spectrally, the radiation is split into two UV channels and a visible (VIS) channel and imaged along the wavelength dimension of the detector array. The spectral resolution of the VIS channel is $0.63 \mathrm{~nm}$. The along-track direction is scanned due to the movement of the satellite. In default "Global" operation mode, five consecutive CCD images, each with a nominal exposure time of $0.4 \mathrm{~s}$, are electronically co-added during a $2 \mathrm{~s}$ interval. The subsatellite point moves about $13 \mathrm{~km}$ during this time interval (Levelt, 2002). The consequence of this design is that the spatial response function of the OMI footprints is not box-shaped but has a peak at the centre of the footprint. This new design, avoiding moving parts, was used in OMI for the first time and is now being used in several new, upcoming satellite missions.

The point spread function (PSF) is defined as the response of the imaging system to a point source, while the telescope field of view (FoV) is defined by the projection of the OMI spectrograph slit on the Earth's surface from the point of view of a CCD pixel. This projection is affected by Fraunhofer diffraction of the imaging optics, which, for a circular aperture, can be modelled using an Airy function. For a rectangular slit, used in OMI, the solution can be approximated by a Gaussian function in two dimensions. The FoV has been determined pre-flight by measuring the intensity response to a star stimulus for all pixels. The response function was measured by exposing the pixels to a point source and rotating the instrument. The sensitivity curve found in this way was fitted to a Gaussian curve, of which the full width at half maximum (FWHM) was reported. This is proprietary information, but the results are summarised here. In the swath (across-track) direction the average peak position for each pixel was determined and fitted to a linear curve to determine the spatial sampling distance for the three channels, which gives the instantaneous FoVs in the across-track direction for individual pixels. For the VIS channel the FoV for the entire swath is $115.1^{\circ}$. The PSF in the across-track direction was not determined (or reported). However, a memo from the OMI Science Support Team from 2005 shows an across-track pixel size estimation from these measurements, where the sizes have been determined by assuming no over- lap between adjacent pixels and computing the distances between the peak positions when imaged on the Earth. This yields sizes in the across-track direction of $23.5 \mathrm{~km}$ at nadir and $126 \mathrm{~km}$ for far off-nadir $\left(56^{\circ}\right)$ pixels.

In the along-track direction the FoV was characterised by tilting the instrument to simulate the movement in the flight direction. The measurements were fitted to a Gaussian curve with variable width for different across-track angles and wavelengths. This width is reported as the FWHM in degrees, which is about 0.95 at nadir and 1.60 at $56^{\circ}$ for the VIS channel. This corresponds to a nadir pixel size in the along-track direction of about $15 \mathrm{~km}$ and a far off-nadir pixel size of about $42 \mathrm{~km}$ when the Gaussian is convolved with a boxcar function whose width is the $13 \mathrm{~km}$ movement of the subsatellite point during the $2 \mathrm{~s}$ exposure.

The instantaneous FoV ( $\mathrm{iFoV}$ ) of the OMI instrument is influenced by a polarisation scrambler, which transforms the incoming radiation from one polarisation state into a continuum of polarisation states (as opposed to unpolarised light). The incoming beam is split into four beams of equal intensity, scrambled, and projected onto the CCD. Since the projections of the four beams are slightly shifted with respect to each other, the polarisation state of the incoming radiation still slightly determines the intensity distribution of the four beams and therefore the $\mathrm{iFoV}$ in the flight direction. The only property which is not dependent on the polarisation state of the incoming radiation is the centre of weight of the four beams. This corresponds to the centre of the ground pixels, which is therefore the only geolocation coordinate that can be determined unambiguously (van den Oord, 2006).

Therefore, centre coordinates are provided in the Level $1 \mathrm{~b}$ data product, but corner coordinates are not. However, for mapping purposes, ground pixel area computations (e.g. for emission estimates per unit area), and collocation, an OMI corner coordinate product was developed, called OMPIXCOR, which is provided online via the OMI data portal (Kurosu and Celarier, 2010). Two sets of quadrangular corner coordinates are provided. One set contains tiled pixel coordinates, which are essentially the midpoints between adjacent centre coordinates, mainly useful for visualisation purposes, as no overlap between pixels is imposed. The other set contains so-called $75 \mathrm{FoV}$ pixel coordinates, which, according to Kurosu and Celarier (2010), correspond to $75 \%$ of the energy in the along-track FoV. The authors assumed a $1^{\circ}$ FWHM for the iFoV to fix a Gaussian distribution and convolved it with a boxcar to model the satellite movement. The area under a Gaussian curve corresponds to about $76 \%$ at FWHM for a normal distribution (exponent of 2); however, the authors claim to have used a super-Gaussian distribution with exponent of 4 for this. In this case the energy contained within the FWHM has increased to about $89 \%$. When this $\mathrm{iFoV}$ is convolved with the boxcar function, the energy within the FWHM will have increased even more. The $75 \mathrm{FoV}$ pixels generally overlap in the along-track direction, since radiation emanating from successive scans enter 
the FoV. The coordinates in the across-track direction, however, are still the half-way points between adjacent pixels.

The application of quadrangular pixel shapes for OMI can become problematic when pixel values are aggregated onto a regular grid (e.g. Level 3 products that are reported on a regular lat.-long. grid). If pixels overlap, which might occur when several orbits are averaged or in the case of $75 \mathrm{FoV}$ pixels, extreme values may be smoothed and reduced due to averaging. A more realistic distribution that preserves mean values can be reconstructed using a parabolic spline surface on the quadrangular grid, resulting in a much better visualisation (Kuhlmann et al., 2014). In cases where values from OMI are compared with those of another instrument, especially one with a higher spatial resolution, the approximate true shape of an OMI pixel is desired. For example, we intend to combine spectral measurements from OMI and MODIS to determine the aerosol direct effect over clouds (de Graaf et al., 2012). To this end, an optimal characterisation of the FoV of the OMI footprint is desired, to optimise the accuracy of the retrieval.

In this paper, the OMI FoV for the VIS channel is investigated by testing various predefined shapes and sizes under various circumstances and determining the maximal correlation between OMI and MODIS reflectances. In Sect. 2, the consistency between overlapping OMI and MODIS reflectances is investigated. A cloud-free scene from 2008 is used to study the FoV under the most optimal circumstances. In Sect. 3, a two dimensional super-Gaussian function with a varying exponent is introduced, which can change shape from a near-quadrangular to a sharp-peaked distribution. Furthermore, the sizes in both along- and across-track directions can be varied. This function is used to define various FoVs, which are investigated for various scenes. The change in FoV is further investigated by looking into the effect of scene and geometry changes during the (varying) overpass times of OMI and MODIS. The conclusions from this study are reported in Sect. 4. The geolocations of the pixels in the UV channels are slightly different from those in the VIS channel. However, the FoV cannot be determined in the same way for the UV, since MODIS measurements do not overlap with these channels spectrally.

\section{Data}

The Aura satellite flies in formation with the Aqua satellite in the afternoon constellation (A-train). Aqua was launched in 2002, to lead Aura in the A-train by about $15 \mathrm{~min}$. The time difference between the instruments within the A-train is controlled by keeping the various satellites within control boxes, which are defined as the maximum distances to which the satellites are allowed to drift before correcting manoeuvres are executed. Therefore, the time difference between OMI and MODIS is variable by up to a few minutes. A major orbital manoeuvre in 2008 of Aqua decreased the distance between the Aura and Aqua control boxes to about $8 \mathrm{~min}$.

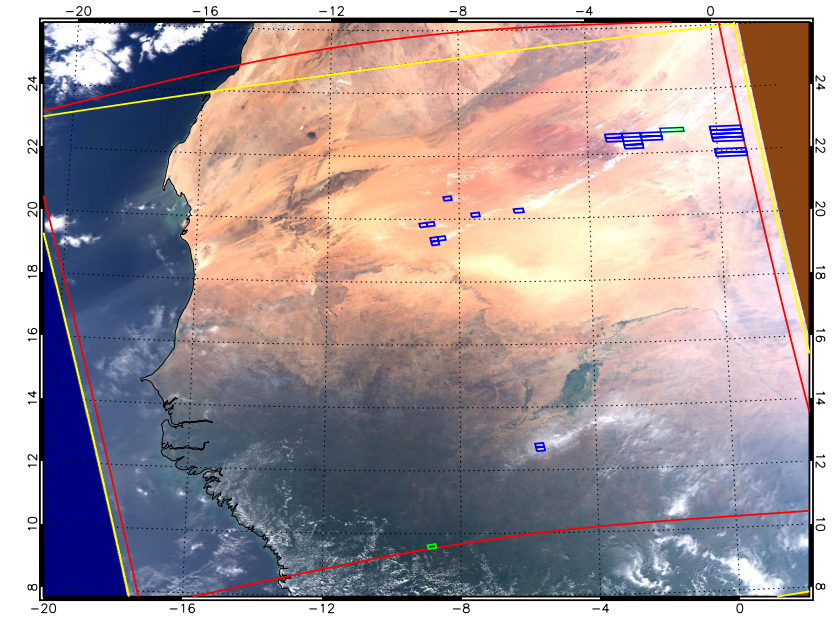

Figure 1. MODIS RGB image of the reference scene on 4 November 2008 at 14:00 UTC (start of the central MODIS granule). The yellow lines indicate the MODIS data granules, and the red lines the considered OMI swath, which was confined to rows 2-57, with the exception of pixels in the row anomaly (see text). The green pixels indicate the darkest (vegetated) and the brightest (cloud-covered) areas in the scene. The OMI reflectance spectra of these pixels are shown in Fig. 2. The blue OMI pixels correspond to the blue-marked points in Fig. 3.

To investigate the correlation between OMI and MODIS observed reflectances, several scenes were selected. One reference scene will be discussed here in detail. It was an almost cloud-free scene over the Sahara on 4 November 2008, around 14:00 UTC (start of the first MODIS granule). At this point in time, the time difference between OMI and MODIS was reduced to $8 \mathrm{~min}$ and around 20-30 s, depending on the pixel row. The differences between the pixel times arise from the fact that MODIS has a scanning mirror, while OMI has no scanning optics but exposes the CCD to different scenes while moving in the flight direction. The scene is visualised in Fig. 1, using MODIS channels 2, 1, and 3 to create an RGB picture at $1 \mathrm{~km}^{2}$ resolution. The MODIS granules are outlined in yellow, while the considered OMI scene is outlined in red. From June 2007 onward, OMI suffered from a degradation of the observed signal in an increasing number of rows, called the row anomaly (OMI row anomaly team, 2012). In November 2008 the anomaly was limited to only rows 53 and 54 for scenes near the Equator. These rows were disregarded in the comparison. In order to stay within the MODIS swath, the OMI swath was further reduced to rows 2 to 57. A total of $7335 \mathrm{OMI}$ pixels are left in the scene.

To compare reflectances from OMI and MODIS, the reflectance measured by OMI is convolved with the MODIS spectral response function. MODIS channel 3 at $469 \mathrm{~nm}$ overlaps with the OMI VIS channel $(350-500 \mathrm{~nm})$. This is illustrated in Fig. 2, where two OMI reflectance spectra from the VIS channel are plotted, together with the normalised 


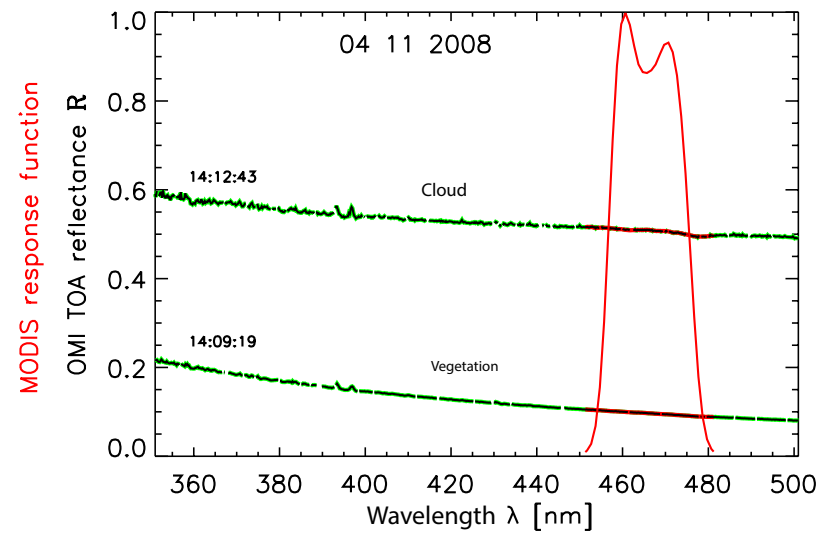

Figure 2. OMI top-of-atmosphere reflectance spectra on 4 November 2008 at 14:09:19 UTC and 14:12:43 UTC of the green pixels in Fig. 1 (black/green), and the normalised MODIS response function of channel 3 (red).

MODIS response function of channel 3 (red curve). The reflectance spectra correspond to the darkest and brightest pixels (at $469 \mathrm{~nm}$ ) in Fig. 1, indicated by the green boxes. The darkest pixel is a vegetated area with an OMI reflectance of 0.0935 , and the brightest pixel is a cloud-covered scene with an OMI reflectance of 0.5040 , both at $469 \mathrm{~nm}$.

All the 7335 OMI pixels in the scene in Fig. 1 were compared to collocated MODIS pixels; see Fig. 3a. Here, all the MODIS pixels that fall (partly) within an OMI quadrangular pixel, as defined by the OMPIXCOR 75FoV corner coordinates, are averaged with equal weight, which is the easiest and quickest averaging strategy. The MODIS reflectances are somewhat lower than the OMI reflectances; a linear fit through the points shows a slope of 0.959 and an offset of 0.0023 . The MODIS reflectances show a Pearson's correlation coefficient $r$ of 0.998 with the OMI reflectances and a standard deviation (SD) of 0.0039. The SD refers to the rms deviation of the measurements to the model fit.

\section{OMI point spread function}

The true FoV of an OMI pixel is expected to resemble a flat-top Gaussian shape. To investigate the OMI FoV, the response at $469 \mathrm{~nm}$ is compared to the MODIS channel 3 signals, weighted using different super-Gaussian functions in two dimensions and checking the change in the correlation and SD between the OMI and MODIS reflectances. A 2-D super-Gaussian distribution is defined by

$g(x, y)=\exp \left(-\left(\frac{x}{w_{x}}\right)^{n}-\left(\frac{y}{w_{y}}\right)^{m}\right)$,

where $x$ and $y$ are the along- and across-track directions, and $w_{x, y}$ are the weights in either direction, defined by
$w_{x}=\frac{\mathrm{FWHM}_{x}}{2(\log 2)^{1 / n}} ; \quad w_{y}=\frac{\mathrm{FWHM}_{y}}{2(\log 2)^{1 / m}}$.

$\mathrm{FWHM}_{x}$ and $\mathrm{FWHM}_{y}$ are the full widths at half maximum in the along- and across-track directions, respectively, defined in this paper by the $75 \mathrm{FoV}$ pixel corner coordinates. The size of the FoV model can be varied to include more or fewer MODIS pixels from neighbouring pixels in the alongand across-track directions by varying $w_{x}$ and $w_{y}$. All size changes are reported relative to $\mathrm{FWHM}_{x}$ and $\mathrm{FWHM}_{y}$.

The shape of the FoV model is determined by the Gaussian exponents $n$ and $m$, which define the "pointedness" of the distribution. In one dimension, $n=2$ corresponds to a normal distribution, $n<2$ results in a point-hat distribution, and $n>2$ results in a flat-top distribution; see the illustration in Fig. 4. Various FoV models are illustrated in Fig. 5. The colours of the square MODIS pixels indicate the relative contribution of that pixel. The different panels show OMI pixels at different rows, to illustrate the change in orientation and number of MODIS pixels that fall inside an OMI pixel when the viewing zenith angle (VZA) changes. Fig. 5a shows the quadrangular OMI pixel, with all MODIS pixels within the OMI corner coordinates having equal weight, while all pixels outside the footprint have zero weight. Figure $5 b$ shows a 2-D flat-top super-Gaussian $(n=m=8)$ shape using the $75 \mathrm{FoV}$ corner coordinates to constrain the FWHM, resembling the quadrangular shape but with smoother edges. Fig. 5c shows a 2-D super-Gaussian distribution, with $n=2$ and $m=4$, which represents the optimal representation of the FoV using a super-Gaussian function. Fig. $5 \mathrm{~d}$ shows a 2-D point-hat super-Gaussian $(n=1, m=1.5)$ distribution, which is the optimal fit of this function when broken clouds are in the scene. Figure $5 \mathrm{e}$ and $\mathrm{f}$ show the weights for pixels which are assumed to be twice as wide or long as the $75 \mathrm{FoV}$ pixels and using a 2-D super-Gaussian distribution with $n=2$ and $m=4$.

The size and shape of the FoV model were varied by changing $n$ from 0.5 to $16, m$ from 1 to 16 , and the FWHM from 0.5 to 3 times the $75 \mathrm{FoV}$ corner coordinates. For each configuration the correlation between the OMI and MODIS reflectances and the SD were determined, using all pixels from the scene in Fig. 1. The correlation change is shown in Fig. 6. The blue solid curve shows the change in correlation for a changing Gaussian exponent and 1. FWHM, i.e. the change in FoV model shape and $75 \mathrm{FoV}$ corner coordinates to constrain the FWHM. In the top panel the change in correlation coefficient $r$ is shown for a changing Gaussian exponent $n$ using the optimal Gaussian exponent found for the across-track direction $m=4$. For this function the optimal Gaussian exponent in the along-track direction is $n=2$. The blue dotted curve shows the goodness of fit $q$ corresponding to each of the correlation coefficients $r$ (the blue solid line). It indicates the probability of a non-random Chi-square fit, which was determined using a constant error for OMI mea- 

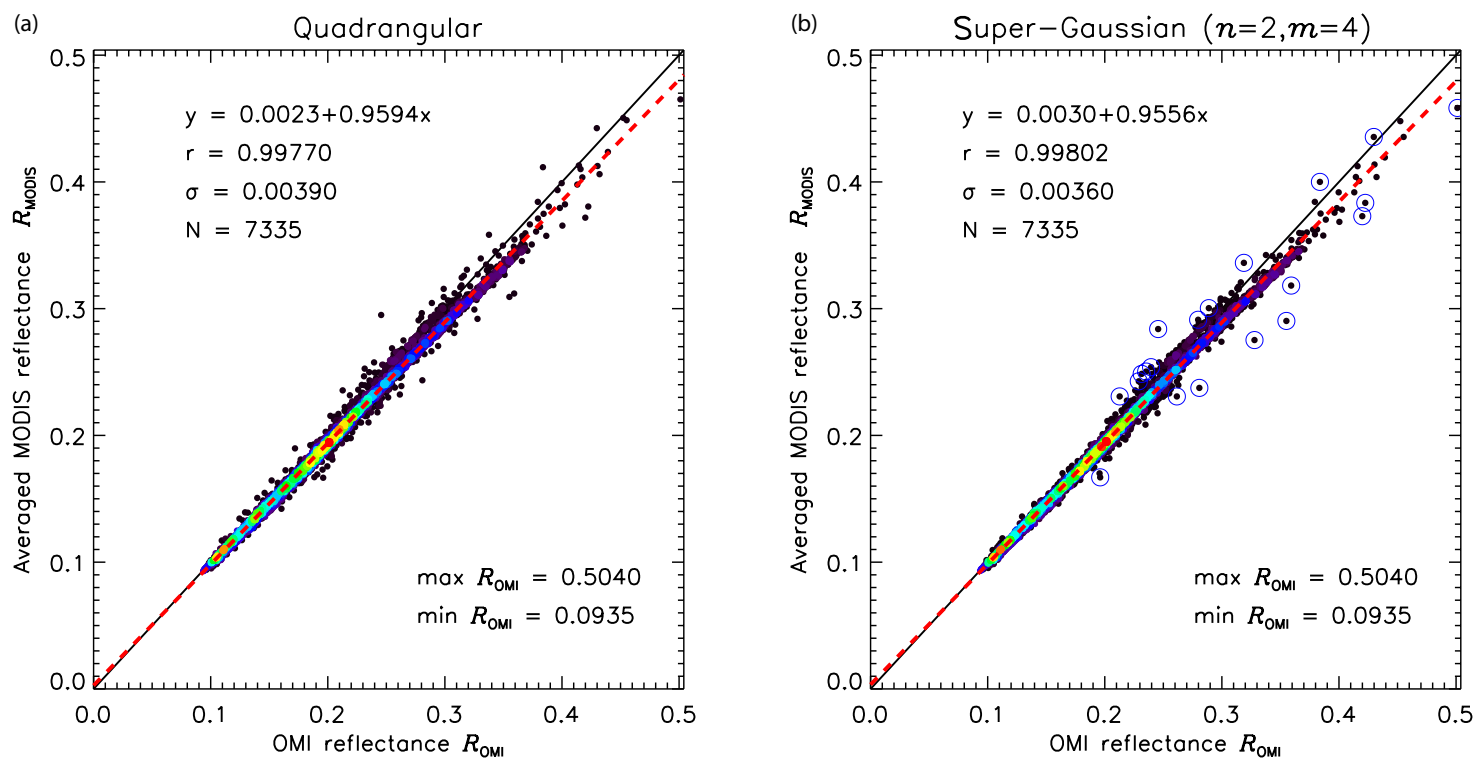

Figure 3. Scatter plot of OMI and MODIS collocated reflectances for the scene in Fig. 1 using quadrangular OMI pixels (a) and optimised super-Gaussian $(n=2, m=4)$ pixels $(\mathbf{b})$. The red dashed line is the linear least-squares fit to the measurements, given by the linear function $\mathrm{y}=a_{0}+a_{1} x$ in the plot. $r$ is Pearson's correlation coefficient, and $\sigma$ the standard deviation of the points to the fitted line. The blue-marked points have the largest $\sigma$ and correspond to the blue OMI pixels in Fig. $1 . N$ is the number of points, and max $R_{\mathrm{OMI}}$ and min $R_{\mathrm{OMI}}$ the maximum and minimum value in the plot, respectively.

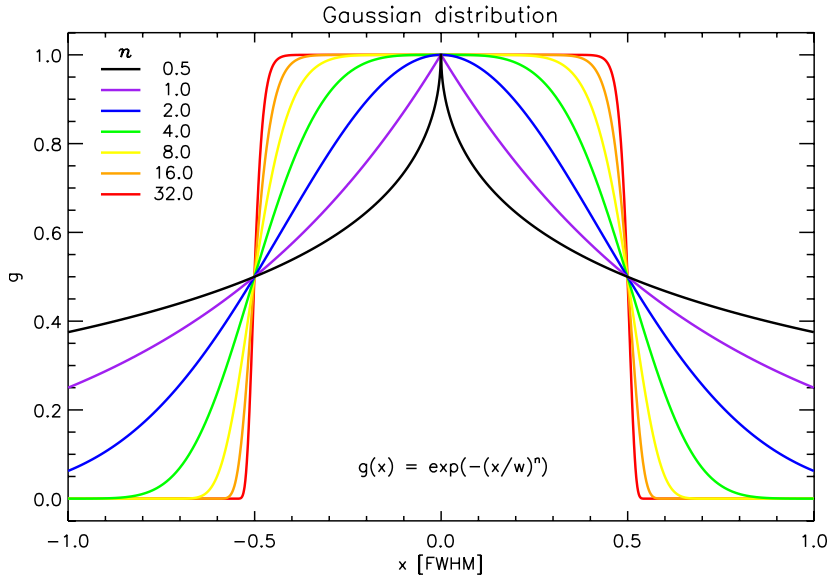

Figure 4. One-dimensional normalised super-Gaussian distribution functions with varying exponents $n$. The normal distribution $(n=2)$ is plotted in blue.

surements, and a constant error for MODIS measurements but weighted by the number of MODIS pixels in each OMI pixel. It shows a reasonably good fit at the optimum $n=2$.

The red line shows the change in correlation when the along-track width is varied. The shown curve is for the optimal Gaussian parameters, $n=2$ and $m=4$, and peaks at 1.0 , meaning that the $75 \mathrm{FoV}$ corner coordinates are the optimal sizes to constrain the FWHM when a super-Gaussian model is used. The lower panel shows the same dependen- cies in the across-track direction. The change of $r$ is shown for changing $m$ (the shown blue solid line is for the optimal Gaussian exponent $n=2$ ) and the red curve is the width in the across-track direction for $n=2$ and $m=4$. The red curve also peaks at 1 , again confirming the $75 \mathrm{FoV}$ corner coordinates, while $m$ peaks at 4 . However, the change for larger $m$ is minimal, meaning that the softness of the edges in the acrosstrack direction make very little difference. Only the goodness of fit $q$ decreases significantly for larger $m$, so $m=4$ can be used as the optimal parameter. These four optimal parameters are also the absolute maximum in the entire parameter space, with $r=0.998$. This is noticeably higher than the correlation when quadrangular pixels are used.

The correlation between the OMI and MODIS reflectances and the SD, when the optimal FoV model for this scene is used, is shown in the Fig. 3b. The SD for the optimal FoV is 0.0036 . The change in SD for different shapes and sizes is not shown, because it is consistent with the change of the reciprocal of the correlation, in the sense that it is minimal when the correlation peaks and can be equally used to find the optimal FoV characterisation in this way.

\subsection{FoV sensitivity}

When a super-Gaussian form is assumed, the optimal superGaussian model parameters for the reference scene are $n=2$ and $m=4$, and the $75 \mathrm{FoV}$ corner coordinates for the Gaussian FWHM. However, the correlation between OMI and MODIS reflectances is not a constant. A number of scenes 

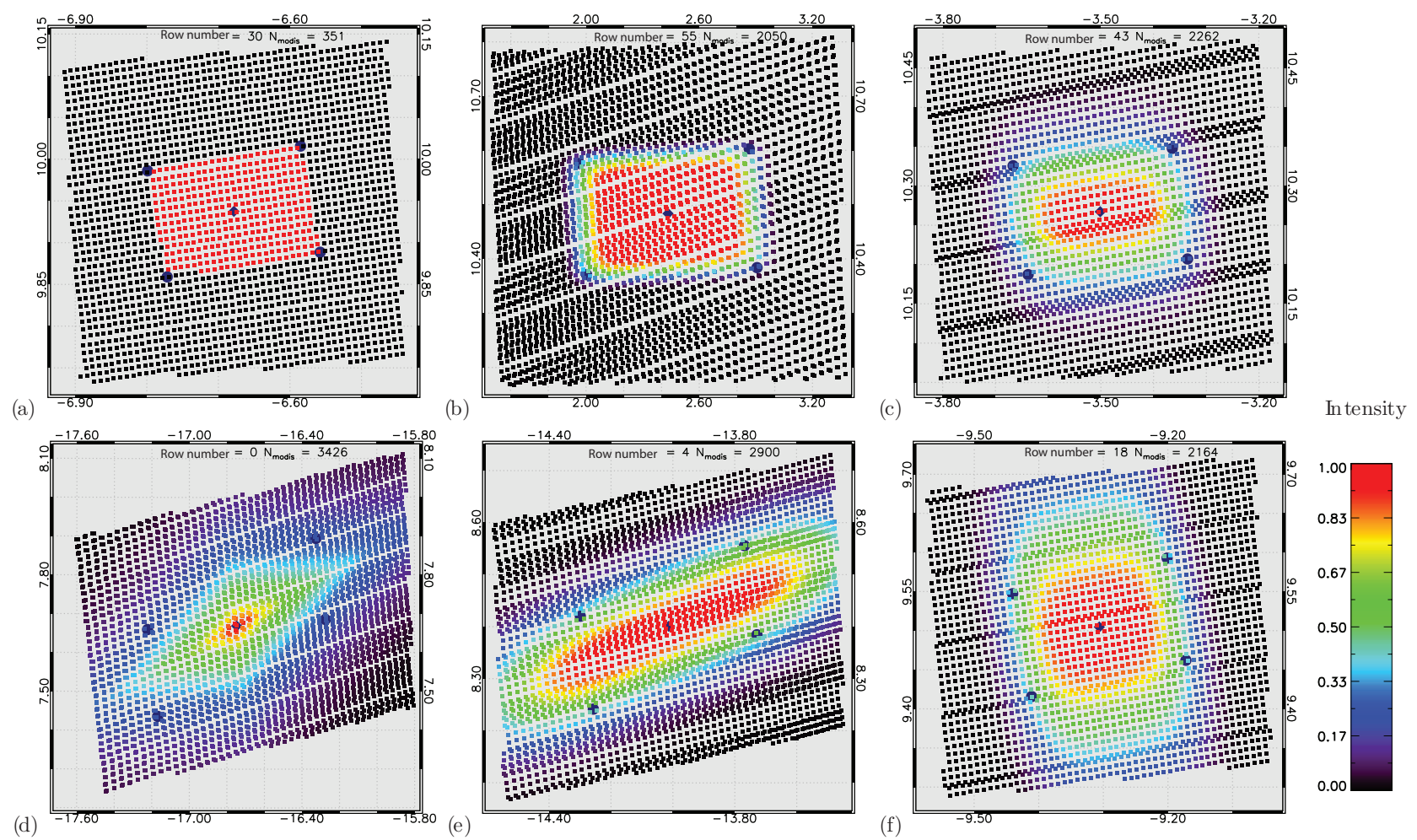

Figure 5. OMI 75FoV corner coordinates (dark-blue-filled circles), with the OMI centre coordinate (dark blue diamond) and collocated MODIS centre coordinates (black and coloured squares). The colours of the squares indicate the weighting of the MODIS pixels as indicated by the colour bar. (a) Quadrangular weighting, with all MODIS pixels within the corner coordinates having equal weights, everything else disregarded; (b) a 2-D flat-top super-Gaussian distribution with exponents $n=m=8$, resembling the quadrangular shape with smoothed edges; (c) a 2-D super-Gaussian distribution with $n=2$ and $m=4$; (d) a 2-D point-hat super-Gaussian distribution with exponents $n=1$ and $m=2$; (e) a 2-D super-Gaussian distribution $(n=2, m=4)$ with twice the width in the across-track direction; (f) a 2-D super-Gaussian distribution $(n=2, m=4)$ with twice the width in the along-track direction. Different OMI row numbers are shown (see panel captions) to show the change in orientation and number of MODIS pixels for different rows.

were investigated to show the change in correlation between OMI and MODIS reflectances in time and space.

First, another cloud-free scene was found over the Middle East on 7 October 2008, starting at 10:20 UTC; see Fig. 7. The time difference between OMI and MODIS is about $8 \mathrm{~min}$ and $34-45 \mathrm{~s}$. This scene is entirely cloud-free over land, and the reflectance ranges from 0.12 over the ocean to 0.41 over the desert. The correlation between the OMI and MODIS reflectances is depicted in Fig. 7b, which displays the same dependencies as in Fig. 6. The highest correlation $(r=0.9977)$ was found for the same super-Gaussian parameters as before, confirming the optimal OMI FoV model. Only the goodness of fit was slightly lower than before, indicating a lower confidence in the correlation.

\subsection{Viewing angle dependence}

Next, a scene over Australia was selected on 11 October 2008 starting at 04:45 UTC; see Fig. 8. The time difference between OMI and MODIS is about 8 min and $35-43 \mathrm{~s}$. This scene has a large cloud-free part, as well as a large cloudy part. Most cloud pixels, indicated by the red rectangles, were not used in the analysis. The correlation between OMI and MODIS for various shapes and sizes is again displayed in the right panel. The maximum correlation for this scene was lower than before, $r=0.9927$, and obtained for a pointhat super-Gaussian distribution with exponents $n=1.5$ and $m=2$, and FWHM corner coordinates. The goodness of fit is significantly lower than before.

One reason for the lower Gaussian exponents of the 2008 Australian scene in the across-track direction is the removal of the pixels at the end of the swath, which were filtered because of the clouds in those pixels. The OMI FoV is dependent on the pixel row, or viewing angle, with wider FoVs at the swath ends. Since most of the cloud pixels are at the swath ends, removing these pixels removes the larger exponents. The viewing angle dependence of the FoV is treated here.

Since the OMI FoV is dependent on the polarisation of the scene, the FoV should also be dependent on the scattering geometry. Furthermore, the diffraction at the edges of the 


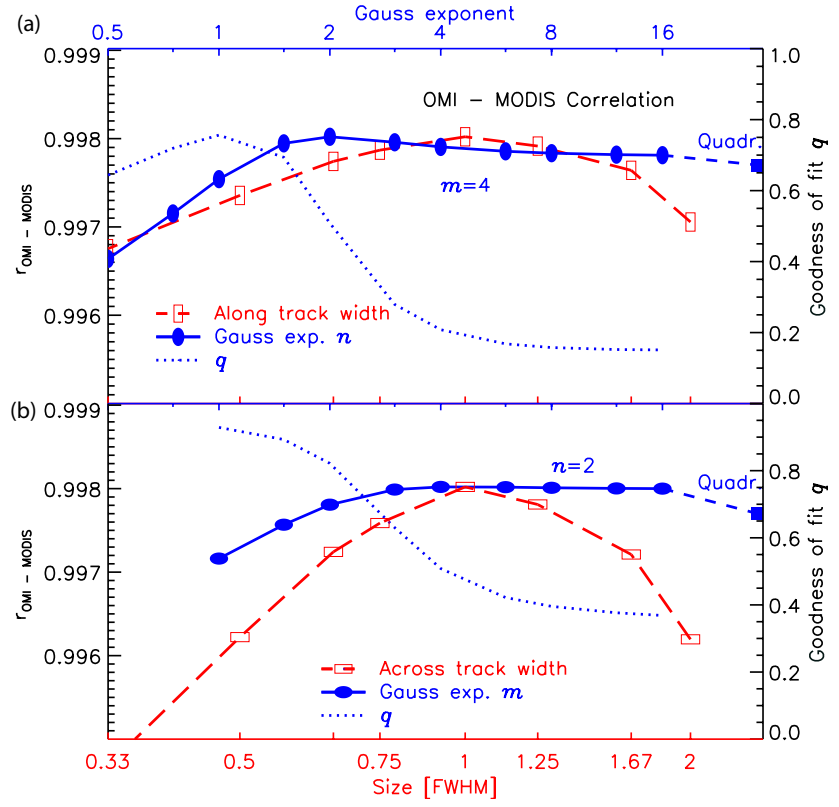

Figure 6. Pearson's correlation coefficient $r$ for OMI and MODIS collocated reflectances in the scene of Fig. 1 as a function of superGaussian shape and size of the assumed FoV. The blue line indicates the correlation as a function of exponent $n$ (a) and $m$ (b) for fixed $75 \mathrm{FoV}$ corner coordinates. The point marked "Quadr." marks the correlation for a quadrangular FoV. The red lines are the relationships for varying pixel sizes when the optimal Gaussian exponents $n=2$ and $m=4$ are chosen. Note that the scales are logarithmic on both $x$ axes.

FoV can be distinctly different for FoVs at nadir compared to those with a large VZA. To investigate this effect, the OMI FoV was characterised using a super-Gaussian function dependent on VZA. For all the scenes described in this paper, the optimal super-Gaussian shape was determined per OMI pixel row, by varying the Gaussian exponent and determining the maximum correlation between OMI and MODIS pixels for each pixel row. Then the optimal exponents were averaged and plotted as a function of pixel row. In this analysis, the $75 \mathrm{FoV}$ pixel sizes were used to reduce the number of variables and because the above analysis showed that the $75 \mathrm{FoV}$ corner coordinates are good indicators of the pixel sizes for Gaussian shapes. The result is shown in Fig. 9. The super-Gaussian exponents are rather wildly fluctuating, because they have a limited sensitivity near the optimum, especially $m$. Averaging over the scenes reduces this but is somewhat arbitrary. In Fig. 9 a boxcar average over five neighbouring points is shown as well.

Still, some change in Gaussian exponents can be observed as a function of VZA. The Gaussian exponent in the acrosstrack direction $m$ changes from around 3-4 at nadir to about 7 at far off-nadir. Also $n$ is VZA dependent, changing from about 1.5 at nadir to more than 2 at the swath edges. The reason for the increasing exponents towards the swath edges is the pixel size increase towards the swath edges. The pixel sizes are shown for reference. FoVs at larger VZA are much wider, changing the optimal super-Gaussian that fit the FoV. Furthermore, as observed before, the diffraction at the edges of the FoV will be different at larger viewing angle.

\subsection{Scene dependencies}

The smaller Gaussian exponents for the 2008 Australian scene (Fig. 8) are only partly explained by the VZA dependence. The Gaussian exponent $n<2$ indicates a pointhat super-Gaussian distribution in the along-track direction, which is, as Fig. 5e shows, a distribution that is physically unlikely. For this scene, the super-Gaussian function is apparently not a good representation of the OMI FoV. The reason for this mismatch is broken cloud fields in the scene, which change the scene reflectance between overpasses of Aqua and Aura. Scene dependencies will be investigated below.

The overpass time between Aqua and Aura changed in 2008, when a correcting manoeuvre brought OMI closer to MODIS. To illustrate the effect, another Sahara cloud-free scene in the beginning of 2008 was selected, when the manoeuvre had not yet been performed; see Fig. 10. The time difference between the instruments for this scene is as large as around $14 \mathrm{~min}$, up to $16 \mathrm{~min}$ and $26 \mathrm{~s}$. In this case, the highest correlation is found for a super-Gaussian distribution with exponents $n=1.5$ and $m=2$, which is again a pointhat super-Gaussian distribution. Similarly, when the shape is fixed to the optimal Gaussian exponents, the highest correlation is found for pixel sizes that are wider than the $75 \mathrm{FoV}$ corner coordinates; see the red curves in Fig. 10. This is different from the reference scene in Fig. 1. The maximum correlation for this scene is $r=0.982$, which is lower than for the reference scene, in December 2008. The goodness of fit $q$ shows much lower values, showing the difficulty with the used FoV model to correlate the OMI and MODIS reflectances. Apparently, the time difference between Aqua and Aura of $15 \mathrm{~min}$ makes a comparison between the two instruments much more challenging, even for almost-cloudfree scenes. It is unlikely that the OMI FoV has changed much between January and December 2008. Furthermore, a cloud-free Sahara scene in 2006 (31 January 2006, around 13:55 UTC, not shown) showed the same lower correlation, peaking for the same Gaussian exponents.

The effect of changing scenes between overpasses can be illustrated by looking at the pixels with the highest SD between the OMI reflectances and the average collocated MODIS reflectances. Even for a scene after 2008, when the overpass time difference is reduced to about $8 \mathrm{~min}$, the retrieved top-of-atmosphere (TOA) reflectance can change significantly during this time in the case of broken clouds. The pixels with the highest SD for the reference scene were marked blue in Fig. 3b. The marked points correspond to the blue coloured OMI pixels in Fig. 1, which are the areas 

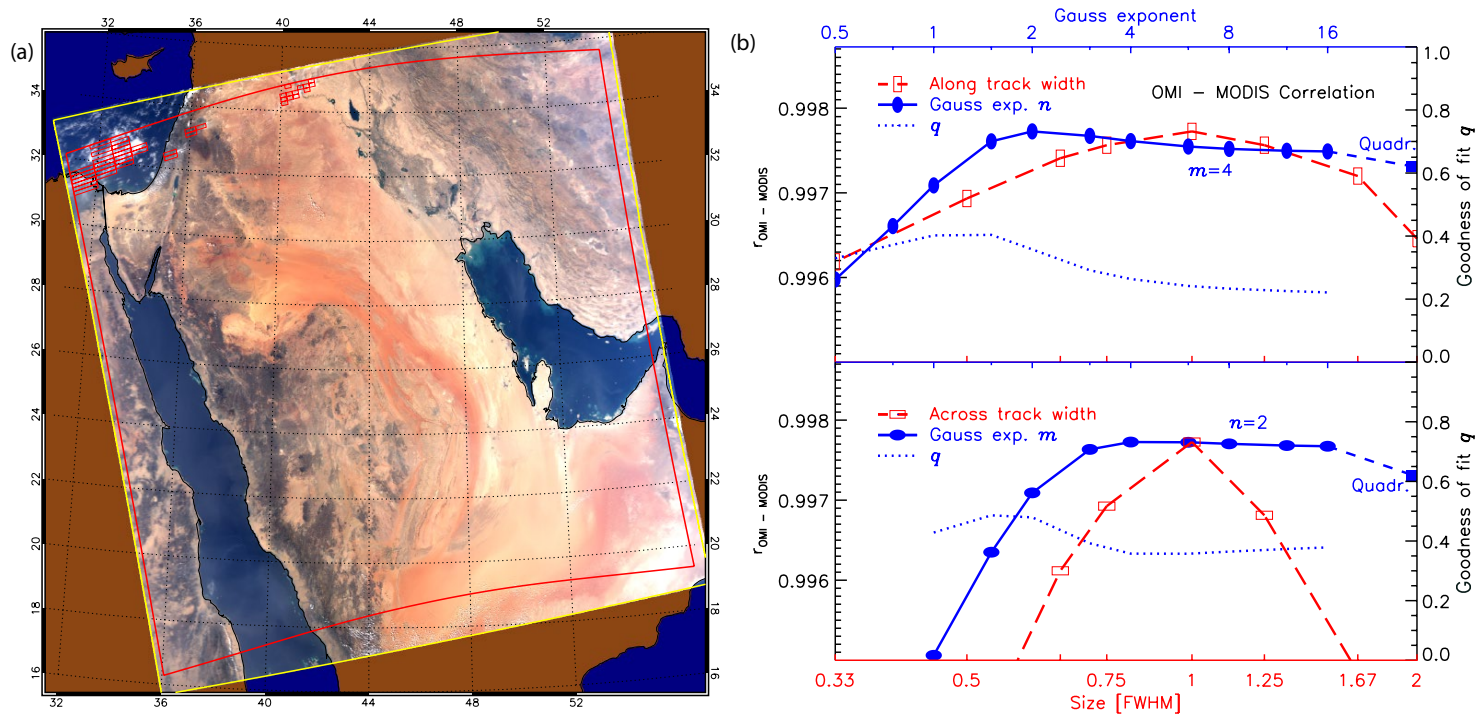

Figure 7. (a) MODIS RGB scene on on 7 October 2008 at 10:20 UTC over the the Middle East. Yellow and red lines as in Fig. 1, while the individual red OMI pixels are cloud pixels that were manually discarded. (b) Dependence of Pearson's correlation coefficient $r$ between the OMI and MODIS observed reflectance for the scene in the left panel as a function of super-Gaussian shape and size, as in Fig. 6. The optimum in this case was found for Gaussian exponents $n=2$ and $m=4$, and $1 \times 75 \mathrm{FoV}$ corner coordinates in both directions.
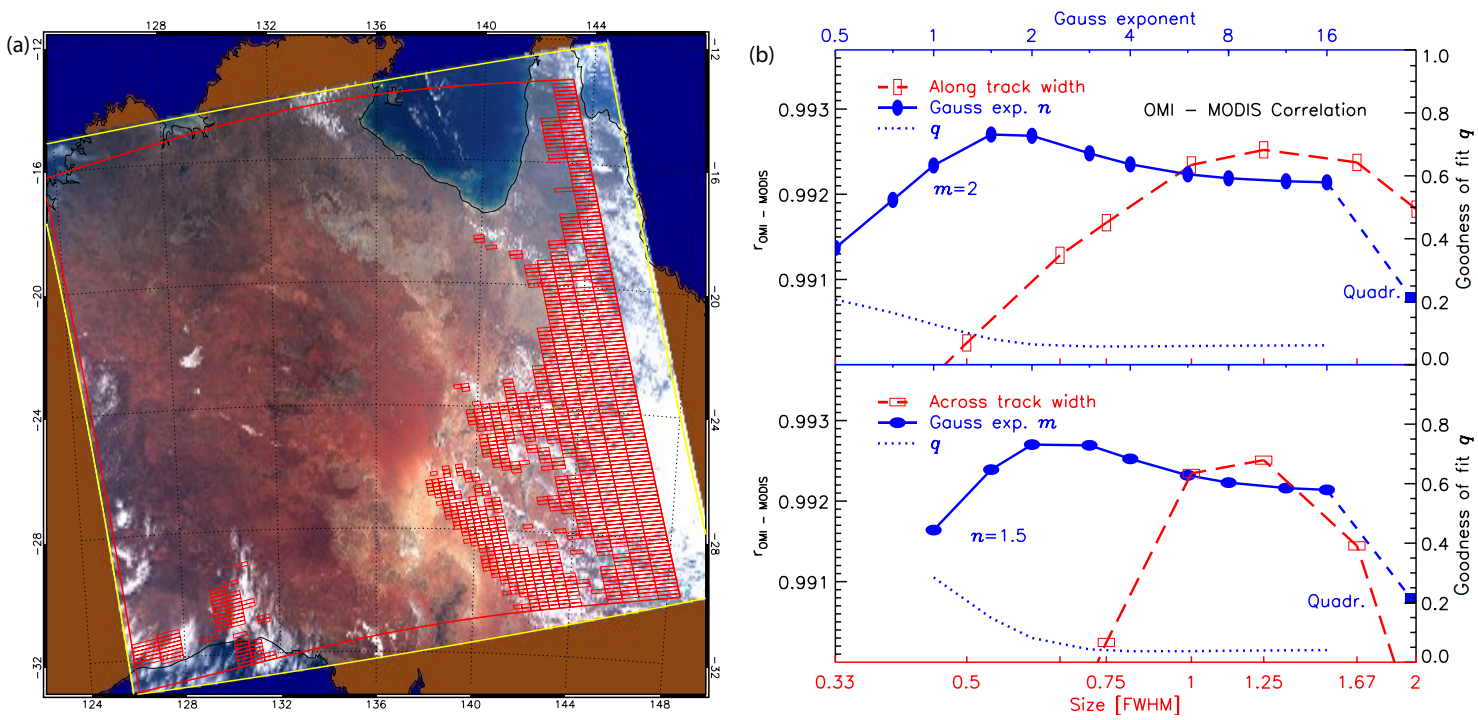

Figure 8. Same as Fig. 7 but on 11 October 2008 at 04:45 UTC over Australia. The optimum in this case was found for Gaussian exponents $n=1.5$ and $m=2$, and $1 \times 75 \mathrm{FoV}$ corner coordinates in both directions. A fit of Gaussian exponents $n=2$ and $m=4$ is best for slightly larger pixels $(1.25 \times 75 \mathrm{FoV}$, red line $)$.

where the scene contains broken cloud fields. In the few minutes between Aqua and Aura overpasses these clouds change shape and position, changing the average reflectance in a pixel when the cloud fraction is changed.

This is the main reason for the small optimal superGaussian exponent for the 2008 Sahara scene (Fig. 10) and the Australian scene (Fig. 8): due to scene changes during the different overpass times, the observed overlap function deviates from the true FoV, which closely resembles a Gaussian or flat-topped Gaussian. Instead a more point-hat distribution with wider wings is found. The centre coordinates have the relative highest correlation, albeit lower than before, while the correlation becomes smoothed over a larger area, giving the tails of the function a higher correlation than for the true FoV. 

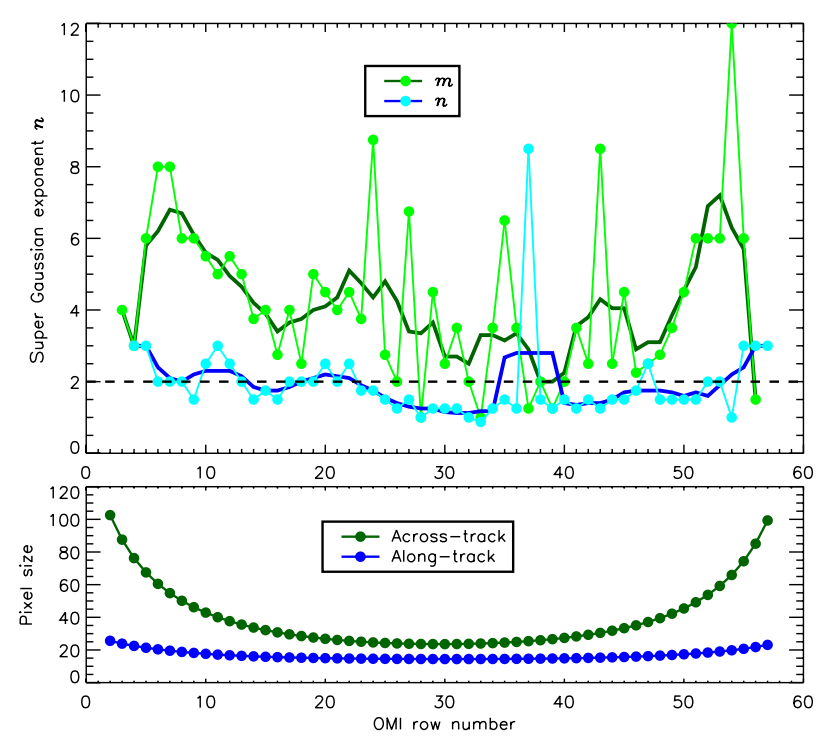

Figure 9. Super-Gaussian exponents $m$ and $n$ as a function of OMI pixel row, averaged over all scenes introduced in this paper. The FWHM was fixed to the $75 \mathrm{FoV}$ pixel sizes, shown in the lower panel, to determine the optimal exponent. The fat lines are boxcar averages using five points.

\subsection{Accuracy of combining OMI and MODIS}

The optimal overlap function for MODIS pixels within an OMI FoV can now be determined for practical purposes, i.e. mixed scenes with ocean, land, and clouds. This is needed to determine the accuracy that can be expected when $\mathrm{OMI}$ and MODIS measurements are combined to reconstruct the reflectance spectrum for the entire shortwave spectrum. To determine the accuracy, the correlation between collocated OMI and MODIS reflectances and the SD was determined by comparing the instruments for the scene shown in Fig. 11. This scene was taken on 13 June 2006, starting on 13:33 UTC, when the time difference between the instruments was about $15 \mathrm{~min}$. The scene contains a mixture of land and ocean scenes, with and without clouds, and also smoke from biomass burning on the African continent. Only OMI rows 10-50 were processed, which will often be the case to avoid problems with large pixels or extreme viewing angles. The optimal correlation was found for superGaussian exponents $n=1$ and $m=1.5$, and $75 \mathrm{FoV}$ corner coordinates (not shown). The low Gaussian exponents can again be explained from the presence of clouds that change the scene between the overpasses, and the exclusion of wide pixels at the swath edges. The correlation between the OMI and MODIS reflectances using this shape is shown in the right panel of Fig. 11. Obviously, the correlation is a lot lower than for cloud-free scenes $(r=0.964)$. The SD is 0.0371, which must be taken into account when OMI and MODIS reflectances are compared or combined. Furthermore, the slope of a linear fit between the OMI and MODIS reflectance is 0.941 , which is smaller than that for cloud-free scenes, which showed about $4 \%$ difference. This larger range in reflectances for cloud scenes apparently offsets the difference between the instruments even further.

\subsection{Geometry differences}

The 4-5\% difference between OMI and aggregated MODIS reflectances at $469 \mathrm{~nm}$ (Fig. 3) can be governed by changes in viewing and solar conditions between OMI and MODIS. Since the optics and subsatellite points differ for both instruments, the viewing angles are slightly different, even if the satellites roughly follow the same orbit. More importantly, since Aura is always behind Aqua, the solar zenith angle for OMI is always different from that of MODIS.

To investigate the effect of the differences in scattering geometry on the measured TOA reflectance, a cloud-free Rayleigh reflectance was modelled for each OMI pixel in the reference scene in Fig. 1. Each pixel was simulated twice, once using the OMI scattering geometry and once using an average MODIS scattering geometry. In this way the expected reflectance difference can be determined due to the difference in overpass time, keeping all else the same. To determine the average MODIS reflectance, the simulated radiances were averaged over the OMI footprint using the optimal flat-top Gaussian distribution with $n=2$ and $m=4$, as was determined for this scene (Fig. 6). The average radiance was then divided by the cosine of the solar zenith angle of the MODIS pixel which is closest to the centre of the OMI pixel. In this way, the most representative solar zenith angle is used to normalise the radiances. A realistic surface albedo was taken for each pixel, in order to make the model results comparable to the observations. The surface albedo database used was the Terra/MODIS spatially completed snow-free diffuse bihemispherical land surface albedo database (Moody et al., 2005). The monochromatic calculations were performed at $469 \mathrm{~nm}$, using a standard Rayleigh atmosphere (Anderson et al., 1986) reaching to sea level and an ozone column of 334 DU. The results are shown in Fig. 12.

The reflectance ranges from about 0.085 to 0.28 , depending on the surface albedo, which is smaller than the observed reflectances (cf. Fig. 3b). This is mainly due to the clouds in the scene which are not simulated. The simulated OMI reflectances are larger than the simulated MODIS reflectances due to different geometries, like the observations. There is a small dependence on VZA, as shown in Fig. 12b, where the relative differences between the OMI and MODIS reflectances are plotted as a function of either reflectance, to highlight the change for changing VZA (in colours). However, the difference between the simulated OMI and MODIS reflectances, with a slope of 0.9965 and an offset of -0.001 , is much smaller than between the observations. Therefore, we conclude that geometry differences between OMI and MODIS introduce differences of less than $1 \%$ and cannot explain the observed slope between OMI and MODIS re- 

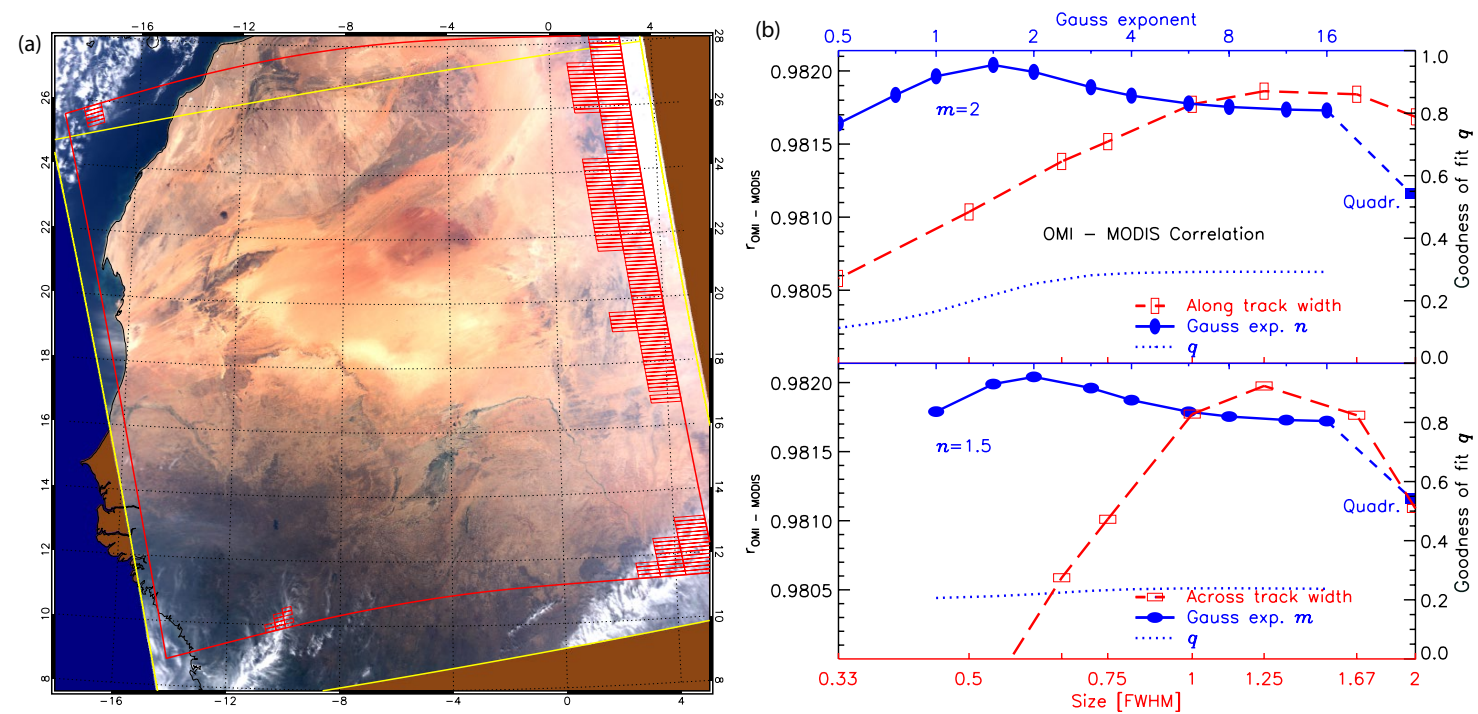

Figure 10. Same as Fig. 7 but on 7 January 2008 at 13:45 UTC over the Sahara. The optimum in this case was found for Gaussian exponents $n=1.5$ and $m=2$, and $1 \times 75 \mathrm{FoV}$ corner coordinates, or $n=2$ and $m=4$, and $1.25 \times 75 \mathrm{FoV}$ corner coordinates in both directions.
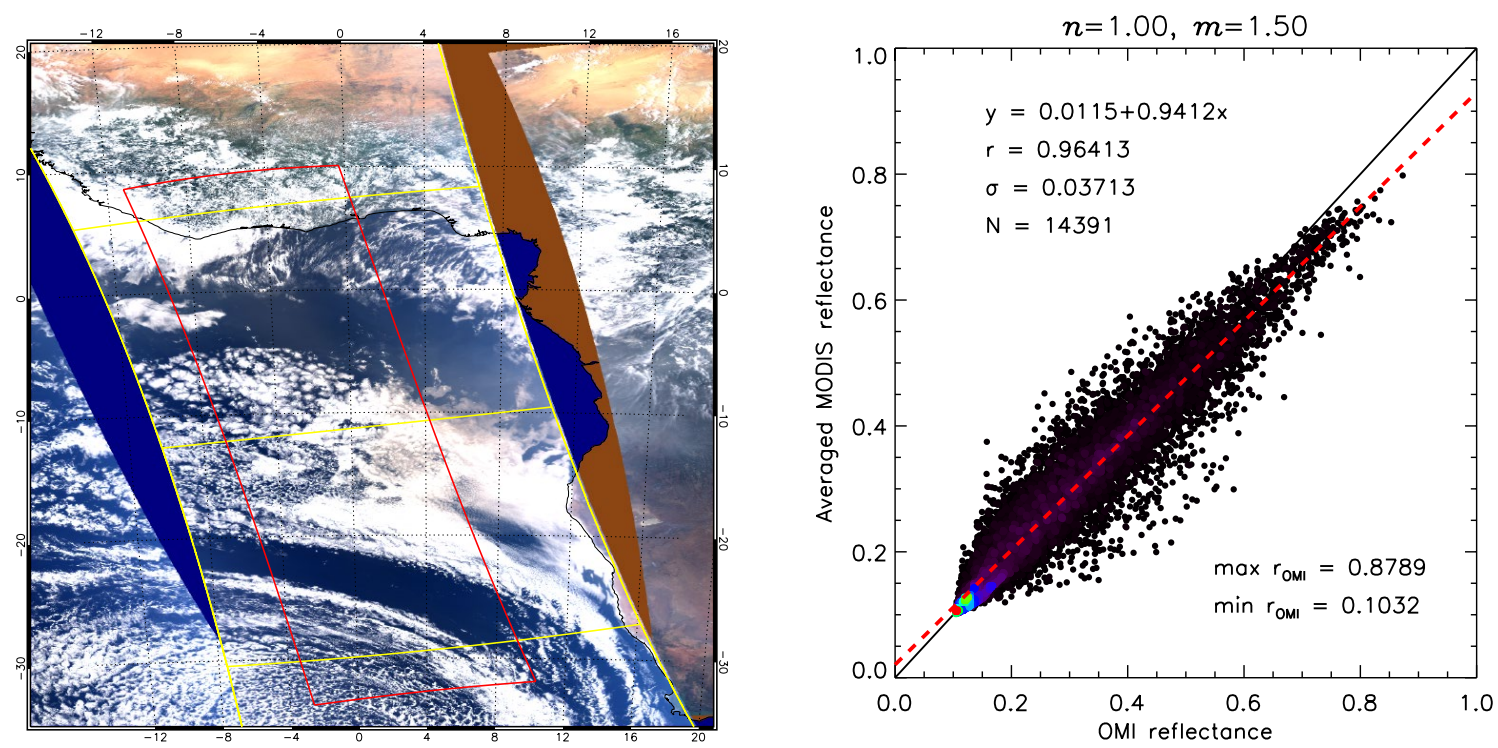

Figure 11. MODIS RGB image on 13 August 2006, around 13:33 UTC (lower part of the image). The yellow lines indicate the MODIS data granules, and the red lines the considered OMI swath, which was from rows 10-50. The optimal correlation between OMI and MODIS for this scene was found for Gaussian exponents $n=1, m=1.5$ and $75 \mathrm{FoV}$ corner coordinates. The correlation for this pixel shape is shown in the right panel.

flectances. Most likely, calibration differences are causing the difference between the observed reflectances. The simulated correlation and SD are also notably better than for the observed scene. As noted before, clouds have the largest impact on the correlation between the observed reflectances of a scene.

\section{Conclusions}

The correlation between OMI and collocated MODIS reflectances was determined, to intercompare the performance of the instruments and to find the FoV of the OMI footprint. MODIS channel 3 at $469 \mathrm{~nm}$ overlaps with OMI's visible channel, and the signals can be compared when the reflectance signal of OMI is multiplied with the MODIS spectral response function, and MODIS reflectances are aggregated over the OMI footprint. 

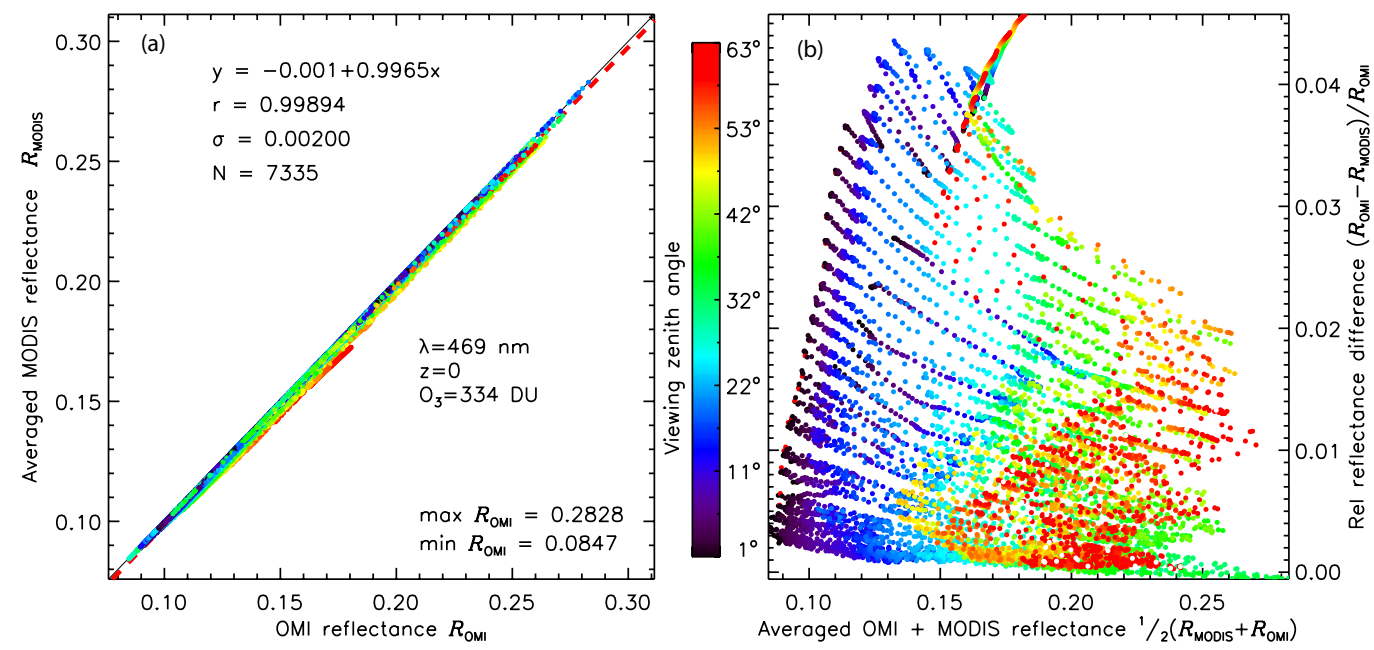

Figure 12. (a) Simulated clear-sky reflectances for the reference scene in Fig. 1 using OMI scattering geometries ( $x$ axis) and MODIS geometries ( $y$ axis). The colours indicate the OMI viewing zenith angle of each simulated pixel. The reflectances were simulated at $469 \mathrm{~nm}$, for a standard atmosphere reaching to sea level and an ozone column of 334 DU. The surface albedo was varied according to a database (see text). The underlying red dashed line shows the linear fit to the simulations. (b) Same data as in the left panel but plotted as the relative difference between the OMI and MODIS reflectances.

Due to the design of the OMI CCD detector array and the optical path, the footprint of OMI is not quadrangular and light from successive scans enters the OMI FoV. The shape and size of the FoV was determined for a cloud-free scene, to eliminate, as much as possible, scene changes due to the different overpass times of Aura and Aqua. Assuming a superGaussian shape with variable exponents and FWHM, the best characterisation of the OMI FoV was found for exponents $n=2$ and $m=4$, and $1 \times 75 \mathrm{FoV}$ corner coordinates to constrain the FWHM.

The OMI FoV changes as a function of viewing angle. When the FWHM are fixed, the Gaussian exponent ranges from about 1.5 at nadir to more than 2 at the swath edges, while $m$ ranges from about 3 to 7 . This is mainly due to the increase in pixel size for off-nadir angles. Furthermore, the diffraction at the FoV edges is viewing angle dependent, and the OMI FoV is dependent on polarisation, due to the presence of a polarisation scrambler in the OMI optical path.

The OMI-MODIS overlap function is scene dependent. In particular, for larger time differences between the Aqua and Aura overpasses, the optimal overlap function shape is found for smaller Gaussian exponents and wider overlaps. When the scene changes between overpasses, the signal is spread over a larger area, centred around the centre coordinate. Therefore, a more optimal overlap function is found for a point-hat distribution with wider wings. This is especially true for cloud scenes, which are most frequent. The correlation decreases and the SD increases when clouds are in the scene, and this can be used as an indication of the expected accuracy of a comparison between OMI and MODIS reflectances. For a scene with broken clouds over both land and ocean in 2006, optimal Gaussian exponents of $n=1$ and $m=1.5$ were found. In general, the changes in correlation coefficient are small for small changes of the Gaussian exponents (much smaller than e.g. changes due to time differences). The true OMI FoV is approximated by a superGaussian distribution with exponent $n=2$ and $m=4$, and 75FoV corner coordinates.

The use of non-scanning optics like those of OMI will be continued in new instruments, in particular TROPOspheric Monitoring Instrument (TropOMI) on Sentinel-5 (Veefkind et al., 2012), to be launched in 2016. For TropOMI, a cloud masking feature is anticipated from Visible Infrared Imaging Radiometer Suite (VIIRS) on Suomi-NPP (Schueler et al., 2002). Sentinel-5P will fly in "loose formation" with SuomiNPP, with expected overpass time differences of about $5 \mathrm{~min}$. The results from this study are relevant for that mission, since such an overpass time difference will significantly change the overlap function between TropOMI and VIIRS, and affect the accuracy of a cloud mask from VIIRS. High-resolution VIIRS measurements can be used in the way presented in this paper to study and characterise the TropOMI FoV and the accuracy of the cloud mask.

\section{Data availability}

The research described in this paper was performed with OMI data, freely available at the Goddard Earth Sciences Data and Information Services Center (http://disc.sci.gsfc. nasa.gov/Aura/data-holdings/OMI), and MODIS data, freely available at NASA's Goddard Space Flight Center (http: //modis.gsfc.nasa.gov/data/). 
Acknowledgements. This project was funded by the Netherlands Space Office, project no. ALW-GO/12-32. Three anonymous referees are thanked for their constructive remarks on the draft manuscript.

Edited by: N. Kramarova

Reviewed by: three anonymous referees

\section{References}

Anderson, G. P., Clough, S. A., Kneizys, F. X., Chetwynd, J. H., and Shettle, E. P.: AFGL Atmospheric constituent profiles, Tech. Rep. AFGL-TR-86-0110, Air Force Geophysics Laboratory, 1986.

Bhartia, P. K., McPeters, R. D., Flynn, L. E., Taylor, S., Kramarova, N. A., Frith, S., Fisher, B., and DeLand, M.: Solar Backscatter UV (SBUV) total ozone and profile algorithm, Atmos. Meas. Tech., 6, 2533-2548, doi:10.5194/amt-6-2533-2013, 2013.

Bovensmann, H., Burrows, J. P., Buchwitz, M., Frerick, J., Noël, S., Rozanov, V. V., Chance, K. V., and Goede, A. P. H.: SCIAMACHY: Mission Objectives and Measurement Modes, J. Atmos. Sci., 56, 127-150, doi:10.1175/15200469(1999)056<0127:SMOAMM>2.0.CO;2, 1999.

Burrows, J. P., Weber, M., Buchwitz, M., Rozanov, V., LadstätterWeißenmayer, A., Richter, A., DeBeek, R., Hoogen, R., Bramstedt, K., Eichmann, K.-U., Eisinger, M., and Perner, D.: The Global Ozone Monitoring Experiment (GOME): Mission Concept and First Scientific Results, J. Atmos. Sci., 56, 151-175, doi:10.1175/1520-0469(1999)056<0151:TGOMEG>2.0.CO;2, 1999.

de Graaf, M., Tilstra, L. G., Wang, P., and Stammes, P.: Retrieval of the aerosol direct radiative effect over clouds from spaceborne spectrometry, J. Geophys. Res., 117, D07207, doi:10.1029/2011JD017160, 2012.

Fleig, A. J., Bhartia, P. K., Wellemeyer, C. G., and Silberstein, D. S.: Seven years of total ozone from the TOMS instrumentA report on data quality, Geophys. Res. Lett., 13, 1355-1358, doi:10.1029/GL013i012p01355, 1986.

Goddard Earth Sciences Data and Information Services Center: OMI data products and data access, available at: http://disc. sci.gsfc.nasa.gov/Aura/data-holdings/OMI, last access: 1 August 2016.
Goddard Space Flight Center: MODIS data, available at: http:// modis.gsfc.nasa.gov/data/, last access: 1 August 2016.

Kuhlmann, G., Hartl, A., Cheung, H. M., Lam, Y. F., and Wenig, M. O.: A novel gridding algorithm to create regional trace gas maps from satellite observations, Atmos. Meas. Tech., 7, 451467, doi:10.5194/amt-7-451-2014, 2014.

Kurosu, T. P. and Celarier, E. A.: OMIPIXCOR Readme File, available at: http://disc.sci.gsfc.nasa.gov/Aura/data-holdings/OMI/ documents/v003/OMPIXCOR_README_V003.pdf, last access: 6 December 2010.

Levelt, P. F.: OMI Instrument, Level $0-1 \mathrm{~b}$ processor, Calibration \& Operations, in: OMI Algorithm Theoretical Basis Document. Volume I, 2002.

Levelt, P. F., van den Oord, G. H. J., Dobber, M. R., Mälkki, A., Visser, H., de Vries, J., Stammes, P., Lundell, J. O. V., and Saari, H.: The ozone monitoring instrument, IEEE T. Geosci. Remote, 44, 1093-1101, 2006.

Moody, E. G., King, M. D., Platnick, S., Schaaf, C. B., and Gao, F.: Spatially complete global spectral surface albedos: Valueadded datasets derived from Terra MODIS land products., IEEE T. Geosci. Remote, 43, 144-158, 2005.

OMI row anomaly team: Background information about the Row Anomaly in OMI, available at: http://projects.knmi.nl/omi/ research/product/rowanomaly-background.php, last access: 26 October 2012.

Schueler, C. F., Clement, J. E., Ardanuy, P. E., Welsch, C., DeLuccia, F., and Swenson, H.: NPOESS VIIRS sensor design overview, in: Proc. SPIE, vol. 4483, 11-23, doi:10.1117/12.453451, 2002.

van den Oord, G. H. J.: OMI Field of View, OMI Science Team Document RP-OMIE-KNMI-XYZ, Issue draft, January 2006, 2006.

Veefkind, J., Aben, I., McMullan, K., Förster, H., de Vries, J., Otter, G., Claas, J., Eskes, H., de Haan, J., Kleipool, Q., van Weele, M., Hasekamp, O., Hoogeveen, R., Landgraf, J., Snel, R., Tol, P., Ingmann, P., Voors, R., Kruizinga, B., Vink, R., Visser, H., and Levelt, P.: TROPOMI on the ESA Sentinel-5 Precursor: A GMES mission for global observations of the atmospheric composition for climate, air quality and ozone layer applications, Remote Sens. Environ., 120, 70-83, 2012. 